\title{
AN EXPERIMENTAL SETUP FOR INSTRUMENTAL ANALYSIS OF FEMORAL DEROTATION OSTEOTOMY
}

\author{
Auer $\mathrm{C}^{1}$, Kallus $\mathrm{S}^{1}$, Eisenmann $\mathrm{U}^{1}$, Korber $\mathrm{J}^{2}$, Wolf $\mathrm{SI}^{2}$, Dickhaus $\mathrm{H}^{1}$ \\ ${ }^{1}$ Heidelberg University, Medical Biometry and Informatics, Heidelberg, Germany \\ ${ }^{2}$ Heidelberg University, Department of Orthopaedic Surgery, Heidelberg, Germany
}

\section{Christoph.Auer@med.uni-heidelberg.de}

\begin{abstract}
Femoral derotation osteotomy (FDO) is an established surgical procedure to recover physiological gait of patients with torsional bony malalignment. Yet in many cases, therapeutic outcome after functional rehabilitation is well behind expectations. Our investigations have revealed methodical problems of the procedure leading to significant measurement bias and poorly reproducible surgical results. Targeting to improve outcome quality, we developed a prototype system to quantify anatomically relevant parameters in FDO using a bone phantom. Using electromagnetic tracking and geometric reconstruction, we achieved accurate and precise measurement of anatomically relevant parameters. Considerations for intraoperative application promise a successful implementation into a CAS system.
\end{abstract}

Keywords: computer model, gait, osteotomy, femur, electromagnetic tracking, measurement, CAS

\section{Introduction}

Patients with torsional bony malalignment showing gait abnormalities usually receive surgical treatment to recover physiological gait. In specialized centers, these procedures are preceded by sophisticated diagnostics utilizing instrumental 3D gait analysis.

An important surgical standard procedure to treat outwards rotated gait is the femoral derotation osteotomy, which readjusts the hip joint angle. The distal femoral bone is cut through and the lower segment is rotated by the diagnostically specified angle around the bone shaft axis. Finally the segments are mechanically fixed with a support plate. To keep track of the rotation, two parallel $\mathrm{K}$-wires are drilled into the bone above and below the cutting location. The adjusted rotation angle is measured by the surgeon using a Moeltgen goniometer to read the angle enclosed by the K-wires [1].

Therapeutic success monitored after one year often falls well behind expectations, not leading to functional improvement for a significant number of patients. The estimated error of adjustment ranges from 10 to 20 degrees. Furthermore a systematic loss of functional correction is evident [2].

Our preliminary research on possible causes of error by means of computer simulation revealed methodical problems of the procedure that potentially lead to either a false estimation of the adjustment or leave functionally relevant side effects on the leg anatomy out of consideration. Results indicate that unwanted tilt of the (ideally perpendicular) cut plane and manual angle readings from a variable and inconvenient point of view have the most significant impact on surgical results.

We concluded that supporting the intervention with accurate measurement devices is essential to improve the quality of femoral derotation osteotomy.

This work investigates the simulation results in a real world scenario with the aid of a tracking system, aiming to provide a prototype measurement setup for intraoperative usage in a CAS system.

\section{Methods}

In order to accomplish our objective, we developed a data acquisition system and protocol to take and record measurements from bone phantoms specifically modified for the purpose of femoral derotation (fig. 1). Considering intraoperative application in a future stage, we chose an NDI Aurora electromagnetic (EM) tracking system to acquire accurate 6-DoF data samples. Small sensors and easy mountability proved suitable for the constrained accessibility of the area of operation. Precautions were taken to avoid electromagnetic induction in the working

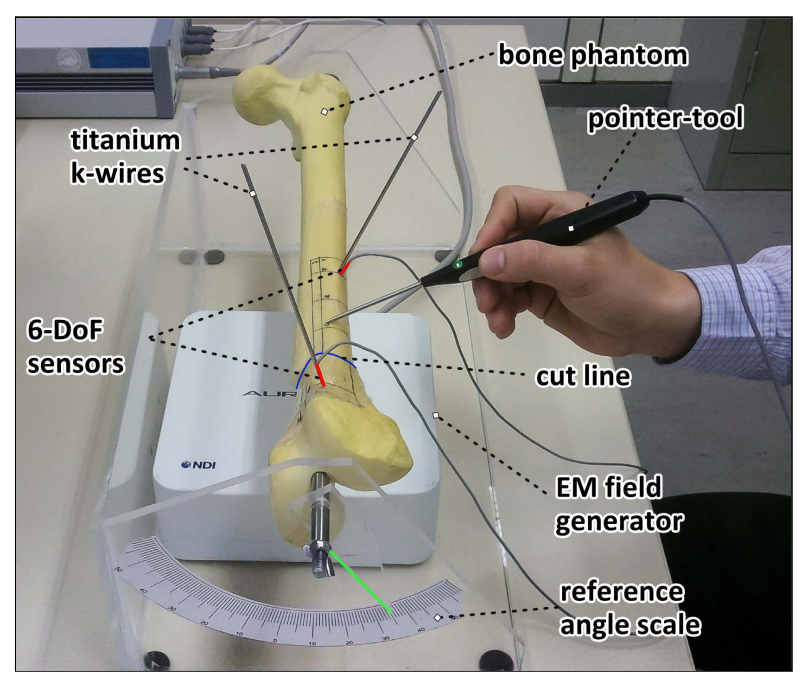

Fig. 1: Experimental setup. A pointer tool is used to take samples from bone surface. The analog angle scale on the bottom end shows the true adjustment angle (reference). 
volume that may interfere with the measurement. Offline analysis was subsequently accomplished in Matlab to reconstruct anatomically relevant parameters such as adjusted rotation angle, displacement of the distal bone segment, etc.

\section{Execution}

Prior to data acquisition, the sensors are mounted to the kwires. The exposed bone surface in between is then sampled sparsely with the pointer-tool. By taking additional samples along the boundary of the cut plane, its (often oblique) orientation is characterized. After derotational adjustment of the distal segment, the separated bone segments are screwed tightly together to fix them. The initial and final positions and orientations of the sensors are recorded by the measuring system.

\section{Analysis}

Using numerical optimization, the bone shaft axis is reconstructed. An initial approximation of the shaft orientation is obtained by principal component analysis of the surface samples. Femoral cross sections change shape and size along the shaft, rendering the reconstruction nontrivial. By grouping the sampling points into a number of equally spaced segments along the shaft and then projecting them onto (virtual) planes perpendicular to the shaft, a center point is determined for each segment (fig. 2). A subdivision into 4 to 6 segments delivers best results while retaining enough samples per segment. The bone shaft axis is finally fitted to the calculated center points. Knowing its exact orientation is of central importance for further parameter reconstruction. Including the orientation of the cut plane, calculation of the rotational axis, tilt and displacement of the knee segment is possible. The resulting adjustment angle is defined by the effective enclosed angle of the sensors along the bone shaft axis relative to its initial magnitude prior to adjustment.

Validation of the results is performed by comparing the computed adjustment to an analog angle scale on the experimental setup (see fig. 1) and simulated results from our computer model.

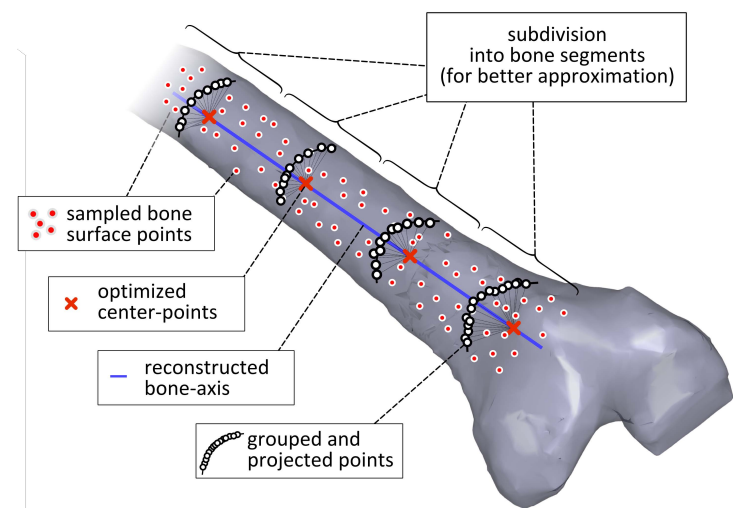

Fig. 2: Reconstruction of bone shaft axis

\section{Results}

Our approach to quantify anatomically relevant parameters in femoral derotation osteotomy by means of a measurement system and geometric reconstruction has shown promising results: We achieved an accurate and precise characterization of the anatomical modifications applied to the bone phantom.

While constraining the measurement locations to the intraoperatively accessible area of operation, reconstructing the adjustment angle was possible for several typical configurations of cut plane, k-wire placement and extends of rotation up to the clinically reasonable maximum of $30^{\circ}$. Deviations to the analog scale remained within $1^{\circ}$, indicating a valid reconstruction of the bone shaft axis. Displacement of the knee joint was observed to behave according to simulation, shifting out of the bone shaft axis by up to $15 \mathrm{~mm}$ in cases of large adjustment angles and tilts of the cut plane nearby $15^{\circ}$.

Preliminary simulation has shown that surgeons may overestimate the adjustment angle by up to $7^{\circ}$ solely due to measurement inaccuracy, which may be further compromised by high observer bias of the manual reading.

The complete process of sensor attachment, measurement execution and recording took 5 minutes on average, with the rigid attachment of the sensors to the k-wires consuming the largest amount of time.

Analysis of the measurement data is available almost instantly after import into Matlab, yet relying on a complete dataset which does not permit real time computation during execution.

\section{Discussion}

The presented prototype measurement setup has shown to provide objective and valid results in a laboratory environment. Due to its considerate design, it can easily complement the established surgical procedure without major modifications or higher invasiveness.

Intraoperative application, however, requires further development to address limitations of the current setup:

High reliability and sufficient accuracy of electromagnetic tracking needs to be guaranteed under surgical conditions with several metal tools involved. This has already been successfully achieved in other surgical domains by suitable calibration methods [3]. Also, additional sensors may be required to allow for passive movement of the patient's leg.

The available, cubic tracking volume with an edge length of $40 \mathrm{~cm}$ leaves little margin for movement or tall patients. Beyond that, a just in time analysis of acquired data is required to provide feedback to the surgeon during execution.

\section{Bibliography}

[1] M. Wager: The Supracondylar Femur Osteotomy for the Correction of a Genu Valgum. Operat Orthop Traumatol 15:387-401, 2003

[2] R. M. Kay, S. A. Rethlefsen et. al: Comparison of Proximal and Distal Rotational Femoral Osteotomy in Children With Cerebral Palsy. J Pediatr Orthop 23(2):150-154, 2003

[3] H. Martinez, D. Mucha et. al: Calibration method for electromagnetic tracked instruments in clinical applications. Int J Comput Assist Radiol Surg 3 (Suppl 1), 111-113, 2008 